\title{
Identifying environmental exposures in pediatric practice: Fundamental validation of a clinical tool
}

\author{
Parneet Jaggi ${ }^{1}$, Irena Buka ${ }^{2}$, Jesus Serrano Lomelin ${ }^{1}$ and Alvaro Osornio-Vargas ${ }^{1 *}$ \\ ${ }^{1 *}$ Department of Pediatrics, University of Alberta Edmonton Clinic Health Academy, 1140587 Avenue NW, Edmonton, AB T6G 1C9, Canada \\ ${ }^{2}$ Clinical Professor of Pediatrics, University of Alberta Director of Children's Environmental Health Clinic (ChEHC) Child Health Clinic, Mother Rosalie Health \\ Services Centre, 231-16930 87 Avenue NW, Edmonton, AB T5R 4H5, Canada
}

\begin{abstract}
Objective: Clinical questionnaires can assist clinicians with the diagnosis, prognosis, and long-term follow up of a disease, or assessing quality of life. However, lack of validity and reliability assessments of the questionnaires before application can lead to bias or outcome misinterpretations. Our Pediatric Environmental Health History (PEHH) is no exception and underwent a fundamental, content validation.

Methods: Expert consensus of a first version of the PEHH (200 questions spanning 9 sections) was collected through a modified Delphi technique. Experts were identified mainly throughout the USA Pediatric Environmental Health Specialty Units. Reaching 90\% agreement (excellent consensus) was predetermined to indicate when to stop the rounds of revisions by the experts. A final version was generated based on expert suggestions, which then underwent a usability and usefulness assessment from parents/guardians $(\mathrm{P} / \mathrm{G})$ and pediatricians, respectively.

Results: Two rounds of review were required for experts to reach excellent consensus on question relevancy for all nine sections. After incorporating experts' suggestions, the final version consisted of 161 refined questions. Upon PEHH completion, $44 \mathrm{P} / \mathrm{G}$ indicated good usability, and majority were satisfied with its length and completion time. Fifteen pediatricians indicated good usefulness after PEHH review, but majority were dissatisfied with its length and anticipated administration time, further suggesting need for a shorter version.
\end{abstract}

Conclusion: Content validity for the PEHH has been established, yielding a relevant and comprehensive set of questions to explore pediatric environmental exposures. Good usability and usefulness have been indicated. Further evaluations such as construct validity and reliability are now, warranted.

\section{Introduction}

Clinical work using validated questionnaires is pivotal in facilitating the identification of suspected environmental exposures and their potential sources in children. Clark, et al., (2010) suggests that a clinical, environmental screening is necessary for children, especially since they display behaviors that place them at greater risk for the exposures [1]. Valuable information obtained from questionnaires can also help clinicians identify aggravating risk factors to address parental/guardian concerns more effectively and further help them make informed decisions.

Although current studies such as one by Fromm, et al., (2010), largely focus on exposure quantification (e.g. biomonitoring) [2], there is still, room for identifying them more widely through questionnaires [3]. Both, human biomonitoring and clinical questionnaires, are necessary to complement one another in identifying and confirming suspected exposures in children. In order to promote data collection of environmental exposures in the clinical setting as well as for health research, relevant tools need to be readily available.

Cumulated experience in dealing with rising numbers of referred respiratory and neurodevelopmental cases at the Children's Environmental Health Clinic at the Edmonton Misericordia Community Hospital, has led to increasing concern about the environment's role. A previous study by Garbutt, Leege, and Sterkel, (2012) has also shown these categories to be amongst the top health concerns for parents [4]. However, when dealing with these cases in relation to the environment, pediatricians feel ill equipped in addressing parental concerns, which leads to low levels of confidence [5-7]. In routine interviews, the majority of pediatricians avoid exploring additional risk factors apart from tobacco smoke, pets, water, lead, and housing [5-7]. Only upon clinical suspicions will few pediatricians question mold, heat, and outdoor/indoor air [5]. Nonetheless, a large proportion have great interest in learning more about environmental health [5-7] and are favorable towards conducting an environmental history with training, as this could allow them to consider possible exposures and appropriate recommendations [8].

We developed the Pediatric Environmental Health History $(\mathrm{PEHH}):$ a questionnaire intended to comprehensively explore environmental exposures in relation to the patient's health history. A full patient evaluation involves using the collected information alongside their medical history. Examples of available questionnaires such as the Green Page [9], the Pediatric Environmental History [10],

Correspondence to: Alvaro Osornio-Vargas, Parneet Jaggi, Department of Pediatrics, University of Alberta Edmonton Clinic Health Academy, 1140587 Avenue NW, Edmonton, AB T6G 1C9, Canada, Tel: (780) 248-5530; E-mail: osornio@ualberta.ca,pjaggi@ualberta.ca

Key words: environment, history, validity, clinical, pediatric

Received: February 07, 2016; Accepted: March 28, 2016; Published: March 31, 2016 
and the Risk Assessment for Lead Exposure: Parent Questionnaire [11] are not as comprehensive. More importantly, they have not undergone validation processes.

Invalid or unreliable questionnaires can lead to clinical bias or outcome misinterpretations. Validation typically depends on the questionnaire's purpose. Terwee (2007) has contributed to the development of explicit quality criteria to assess various forms of validity [12]. Content validity is a fundamental step, required to ensure that the questions are relevant and comprehensive for their specific fields and for the target population [12]. We aimed to use the recommended Terwee quality criteria for content validity to a) gather expert opinion to establish the content for the PEHH, and then b) obtain usability and usefulness feedback from $\mathrm{P} / \mathrm{G}$ and pediatricians, respectively.

\section{Methods}

The PEHH is comprised of questions and sections supported by scientific evidence, clinical experience, and expert opinion, including the World Health Organization (WHO) teaching modules [13]. The initial version of the PEHH consisted of 200 questions spanning 9 sections: Demographics, General Environment, School Environment, Day Care/Day Home Environment, Lifestyle, Infancy/Childhood, and Prenatal Exposures. A large proportion of clinically encountered cases are respiratory (e.g. asthma) and neurodevelopmental (e.g. ADHD, autism, developmental delay), thereby rendering two additional sections: Additional Environmental Factors Affecting Respiratory Symptoms and Neurodevelopmental Symptoms. The number of questions varies from 15 to 35 per section and the majority offer additional space for detailed responses. Question repetition occurs throughout some sections when appropriate. Many sections may also only receive attention depending on their pertinence to the clinical case.

\section{Content validity}

A modified Delphi technique [14] was used to retrieve thorough feedback and consensus on the PEHH content from qualified environmental experts. Experts were not required to suggest/ brainstorm questions of interest as the PEHH questions were generated a priori, hence our technique modification [14]. Experts were identified mainly throughout the USA Pediatric Environmental Health Specialty Units (PEHSUs) network and the WHO; the main promoters of Children's Environmental Health $[15,16]$. Expert eligibility required an MD qualification, at least five years of clinical and/or academic experience in pediatric environmental health, and English-speakers. Our Delphi subjects were "highly trained and competent within the specialized area of knowledge" [17], Our goal was to establish a minimum panel of 10 experts, since a panel of 10-15 individuals is sufficient to provide feedback $[17,18]$.

Participants received a survey with the original PEHH through REDCap [19], which is hosted and supported by the Women and Children's Health Research Institute's Clinical Research Informatics Core" at the University of Alberta. Experts were instructed to provide question-by-question and global appraisals. The former entailed either agreement or disagreement with the relevancy of each question per section, after thorough review. For questions that received disagreement, we requested modification suggestions including additions, rewording, clarifications, or deletions. Percent Agreement (PA), the average percent of experts agreeing with an individual question as relevant, was used to determine the level of consensus achieved per section [20]. Based on Lynn (1986), we chose a PA of $90 \%$ as our criterion for excellent content validity per section [21]. Additional rounds of review were sought if this criterion was unachieved, indicating need for question(s) improvement [21].

The global appraisal involved a holistic assessment of each PEHH section through responding to 4 statements addressing: 1) question relevancy, 2) comprehensiveness, 3) relevance to pediatrics, and 4) whether questions were an accurate reflection of the underlying concept. Response options were coded on a 5-point Likert scale: $1=$ strongly disagree, $2=$ disagree, $3=$ no opinion, $4=$ agree, and $5=$ strongly agree. Each expert was to provide 36 ratings in total. Space was provided for additional narrative feedback, per section. Median ratings were determined for each expert and an overall median rating was derived per global statement.

Expert comments were reviewed by three independent investigators (AO, IB, PJ) and questions/items for the next round were selected using predetermined criteria. Experts were given opportunity to review the PEHH changes and suggest further modifications in the next round. The revisions were reiterated to only those experts who completed round one. They retrieved the survey (modified to accommodate new PEHH questions), a copy of their original responses, and both PEHH versions for reference to their primary suggestions.

\section{Usability and usefulness assessment}

After establishing content validity, parent/guardians were invited to complete a PEHH and a ten- question usability survey, both telephone administered. This survey targeted ease of answering PEHH questions, appropriateness of response options, question comprehension, and respondent burden [22]. Recruitment occurred in the Edmonton Child Health Clinic, Misericordia Community Hospital waiting room. P/G were informed of the study and consent was obtained.

Pediatricians assessed PEHH usefulness through an online, tenquestion survey upon providing consent. Edmonton pediatricians and clinical fellows throughout the Department of Pediatrics, University of Alberta were of target interest. Departmental pediatricians were identified using an academic contact list. Community pediatricians, who are members of the Canadian Pediatric Society Community Section e-forum, were also invited to participate. Given our objective of gathering general usefulness feedback, we aimed to include 10-15 pediatricians. This is considered sufficient depending on the topic of interest [23-26] and was feasible for study purposes. This study was approved by the Faculty of Medicine \& Dentistry, University of Alberta, Health Research Ethics Board.

\section{Statistical analysis}

\section{Content validity}

All data analyses were conducted using STATA version 13, statistical software. Upon determining the PA per section, we used the standard deviation in each round to indicate the convergence of opinion. A lower standard deviation and an increased PA would portray greater convergence of opinion and content improvement, respectively. Median ratings were determined for each global question. Higher median ratings in conjunction to a decreased rating range would indicate more agreement with the global questions and therefore, content improvement.

\section{Usability and usefulness}

The usability survey was conducted among $\mathrm{P} / \mathrm{G}$, aiming to cover the most commonly encountered conditions seen in the Child Health 
Clinic (e.g. Respiratory, Neurodevelopmental, and “Other”). To ensure a diversity in conditions, we aimed to include a minimum of at least ten cases in each category (total of 30 cases). Both of the usability and usefulness survey responses were coded on a scale of 1 (strongly disagree) to 4 (strongly agree). Median ratings for each question were derived after collating the responses. For usability, the responses were analyzed according to condition category. Higher $\mathrm{P} / \mathrm{G}$ and pediatrician ratings indicate greater agreement and satisfaction with the different $\mathrm{PEHH}$ aspects of interest.

\section{Results}

\section{Content validity}

Thirty-two environmental experts were identified throughout the PEHSU regions, and ten throughout the WHO, for 42 total, eligible participants. Of the 42 potential participants, 22 (52\%) agreed to participate. Two declined due to lack of time, and one declined, as his work did not currently involve pediatric environmental health. The remainder did not respond to our invitation. Of those who agreed to participate, we received 12 submissions (55\%). Two of these experts did not provide a complete response set and were contacted for resubmission without success. Our analysis therefore, included 10 complete surveys in round 1. Eight experts were from the USA, one from Canada, and one from Uruguay. The experts represented the following disciplines: pediatric toxicology, preventive medicine, developmental pediatrics, hazard assessment, general pediatrics, clinical pharmacology, and pediatrics from the family medicine perspective. Their experience in

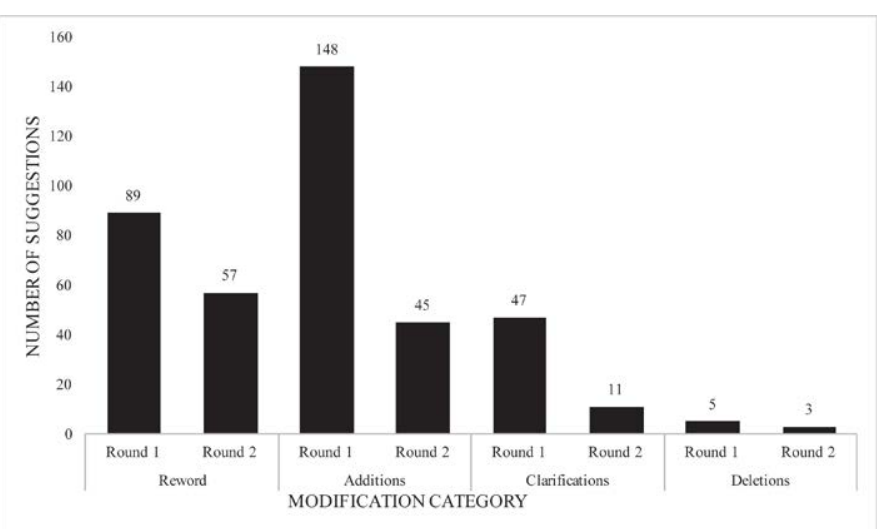

Figure 1. Summary of the total number of suggestions per modification category from rounds 1 ( $\mathrm{n}=10$ experts) and 2 ( $\mathrm{n}=8$ experts). pediatric practice ranged from 5 to 35 years.

\section{Modified Delphi: Round 1 results}

The 10 experts provided a total of 289 suggestions based on the four suggestion categories: re- wording $(\mathrm{n}=89,31 \%)$, additions $(\mathrm{n}=148,51 \%)$, clarifications $(\mathrm{n}=47,26 \%)$, and deletions $(\mathrm{n}=5,2 \%)$ (Figure 1). These involved $55 \%$ of the PEHH questions in total. Since majority $(51 \%)$ of the total suggestions represented additions, this indicated a need to improve PEHH comprehensiveness. The overall nature of these suggested additions were to include more probing questions encompassing the main 'exposure' questions. The PA for the nine sections ranged from $79 \%$ (Lifestyle section) to $94 \%$ (Neurodevelopmental section) (Table 1). All of the global questions had an overall median rating of 4 (agree). Global comprehensiveness had the largest rating range from 2 (disagree) to 5 (strongly agree), suggesting the need to improve PEHH comprehensiveness (Table 2). $\mathrm{PEHH}$ revisions and survey re-iteration was required since 5 sections did not meet excellent content validity [21].

Of the total 289 suggestions provided by the 10 experts, 182 were accepted and 107 were rejected upon team consensus. Rejected suggestions, as per specific criteria were those that would provide redundant information, were not anticipated to enhance understanding of the patient, and were not phrased in Canadian-English. Sixty-seven questions were reworded, 69 added, and 21 deleted, resulting in 248 questions in the new PEHH version, v2 (Figure 2), which underwent subsequent review.

\section{Modified Delphi: Round 2 results}

Only 9 of the original 10 experts participated in round 2. Eight of these experts completed a full survey and the ninth only globally appraised each section. The 8 experts provided a total of 118 suggestions involving 32\% $(\mathrm{n}=79)$ of the PEHH questions (Figure 1). The majority of these suggestions included rewording $(n=58,49 \%)$, and fewer additions were requested $(n=45,38 \%)$ (Figure 1$)$. The PA ranged from $91 \%$ to $98 \%$ (Table 1), indicating excellent content validity and no further need for reiteration [21]. Eight sections improved due to increased PA (Table 1), and the standard deviation decreased for all of the sections, indicating more response convergence (Table 1). Numerical differences in the number of questions per section within each version of the PEHH are highlighted in Table 1. The overall median rating for the global questions remained at 4 (agree) in both rounds, but all ranged between 4 (agree) and 5 (strongly agree) in round 2 (Table 2). Compared to round 1 , the rating range decreased for

Table 1. Expert consensus: Percent agreement (PA) per domain, from surveys round 1 ( $\mathrm{n}=10$ experts) and round 2 ( $\mathrm{n}=8$ experts).

\begin{tabular}{|c|c|c|c|c|c|c|c|}
\hline \multicolumn{4}{|l|}{ Round 1 (Version 1) } & \multicolumn{4}{|c|}{ Round 2 (Version 2) } \\
\hline Domain & Number of Items & $\begin{array}{c}\text { Percent } \\
\text { Agreement }\end{array}$ & $\begin{array}{l}\text { Standard } \\
\text { Deviation }\end{array}$ & Domain & Number of Items & Percent Agreement & Standard Deviation \\
\hline Demographics & 23 & $87 \%$ & \pm 13.03 & General Information & 26 & $95 \%$ & \pm 6.71 \\
\hline General Environment & 29 & $89 \%$ & \pm 9.70 & General Environment & 38 & $92 \%$ & \pm 6.67 \\
\hline School Environment & 16 & $89 \%$ & \pm 11.98 & School Environment & 26 & $95 \%$ & \pm 5.28 \\
\hline $\begin{array}{l}\text { Daycare/Day Home } \\
\text { Environment }\end{array}$ & 17 & $93 \%$ & \pm 9.87 & $\begin{array}{l}\text { Daycare/Day Home } \\
\text { Environment }\end{array}$ & 26 & $98 \%$ & \pm 3.02 \\
\hline Lifestyle & 21 & $79 \%$ & \pm 18.45 & Lifestyle & 20 & $94 \%$ & \pm 7.91 \\
\hline Prenatal Exposures & 32 & $89 \%$ & \pm 9.66 & Prenatal Exposures & 40 & $97 \%$ & \pm 5.45 \\
\hline Infancy/Childhood & 11 & $92 \%$ & \pm 11.58 & Infancy Diet & 17 & $91 \%$ & \pm 10.14 \\
\hline Respiratory Symptoms & 15 & $93 \%$ & \pm 31.15 & Respiratory Symptoms & 22 & $94 \%$ & \pm 6.94 \\
\hline $\begin{array}{l}\text { Neurodevelopmental } \\
\text { Symptoms }\end{array}$ & 36 & $94 \%$ & \pm 31.20 & $\begin{array}{c}\text { Neurodevelopmental } \\
\text { Symptoms }\end{array}$ & 33 & $97 \%$ & \pm 3.59 \\
\hline
\end{tabular}


Table 2. Median global ratings from rounds 1 ( $n=10$ experts) and 2 ( $n=9$ experts).

\begin{tabular}{|c|c|c|}
\hline Global Question & Round 1 & Round 2 \\
\hline & Overall Median Rating (Range) & Overall Median Rating (Range) \\
\hline 1. The questions are overall relevant to the domain & $\begin{array}{c}4 \\
(4-5)\end{array}$ & $\begin{array}{c}4 \\
(4-5)\end{array}$ \\
\hline 2. The questions are overall comprehensive within the domain & $\begin{array}{c}4 \\
(2-5)\end{array}$ & $\begin{array}{c}4 \\
(4-5)\end{array}$ \\
\hline 3. The questions are an accurate reflection of the construct & $\begin{array}{c}4 \\
(3-5)\end{array}$ & $\begin{array}{c}4 \\
(4-5)\end{array}$ \\
\hline 4. The questions are overall relevant to the pediatric population & $\begin{array}{c}4 \\
(4-5)\end{array}$ & $\begin{array}{c}4 \\
(4-5)\end{array}$ \\
\hline
\end{tabular}

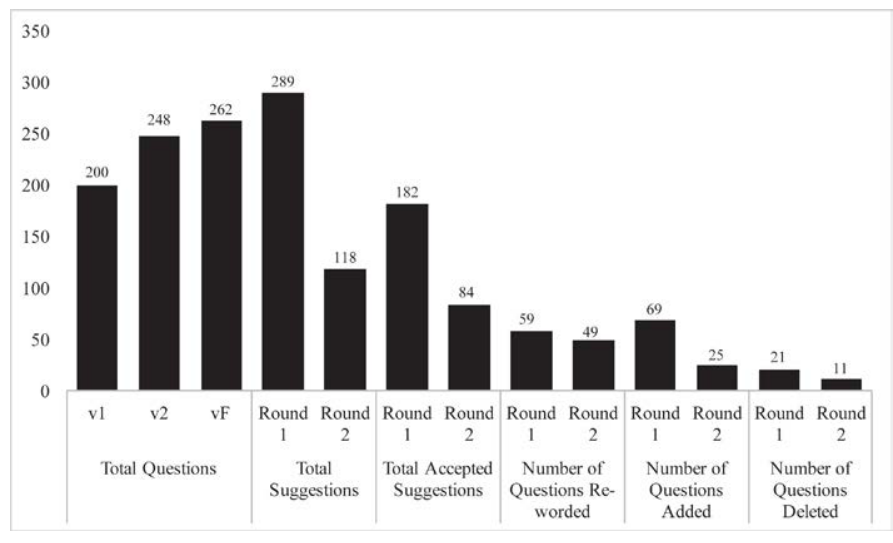

Figure 2. Summary comparison of the number of questions re-worded, added and deleted, and subsequent number of questions in version 1 (v1), version 2 (v2) and Final version (vF).

global comprehensiveness to between 4 (agree) and 5 (strongly agree), suggesting global comprehensiveness improvement. A decreased number of suggested additions, a decreased PA standard deviation per section, and a decreased global rating response range suggest overall, content improvement.

To address round 2 suggestions, we focused on improving question clarity and incorporating any final requests, provided they met the $a$ priori selection criteria. Of the total 118 suggestions, we accepted 84 . Fifty-three questions were reworded, 25 added, and 11 deleted, yielding 262 questions in the final version (vF) (Figure 2). This version was further refined through a 'tiered approach' in order to streamline the exposure assessment questions. Each numerical question represents an independent exposure or "screening question". The subsequent alphabetical questions or "probing questions" permit response expansion if the screening questions are affirmative. This tiered approach resulted in 161 independent questions with an additional 101 subsequent options permitting deeper exploration, if needed.

\section{PEHH usability and usefulness assessment}

Forty-four P/G completed both PEHH and usability surveys. They represented eleven, fourteen, and twenty cases categorized as "respiratory", "neurodevelopmental", and "other" conditions, respectively. Respiratory conditions included asthma and shortness of breath or reduced stamina. Neurodevelopmental conditions included developmental delay, spatial and visual concerns, attention deficit hyperactive disorder, post-traumatic stress disorder, anxiety, depression, bipolar disorder, schizophrenia, and expressive language disorder. "Other" cases included child routine check-ups, dermatitis, otitis media, eczema, fever, acute urinary tract infections, gastrointestinal symptoms, and breastfeeding concerns.

The average age of the $44 \mathrm{P} / \mathrm{G}$ was $35.11 \pm 7.02$ years. The average child age was $6.13 \pm 5.26$ years and the average, questionnaire completion time was $28.89 \pm 6.17$ minutes. According to condition, most of the children in the "other" category were generally younger $(2.47 \pm 2.87$ years $)$ than the respiratory $(10.04 \pm 3.68$ years $)$ and neurodevelopmental ( $9.65 \pm 5.49$ years) patients. The average $\mathrm{P} / \mathrm{G}$ age was also slightly lower for those in the "other" category $(29.81 \pm 6.52$ years) compared to the respiratory and neurodevelopmental categories ( $39.44 \pm 4.62$ and $42.15 \pm 7.46$ years, respectively).

The median ratings were either 3 or 4 for each usability question (Table 3). Only one P/G with a child in the "other" category was dissatisfied with her devoted time, the length of the PEHH, and overall experience. Two participants felt that the PEHH did not offer them an environmental learning experience and did not do a good job of exploring their environment. In the "respiratory" category, one $\mathrm{P} / \mathrm{G}$ was dissatisfied with $\mathrm{PEHH}$ length. In the "neurodevelopmental" category, $2 \mathrm{P} / \mathrm{G}$ did not feel that the PEHH offered them a learning experience.

Our pediatrician population was predominantly from the department, complemented by those from the Canadian Pediatric Society. We received fifteen complete surveys within our study time frame, of which 9 were from departmental pediatricians, one from a clinical fellow, and 5 from community pediatricians. These participants represented pediatric disciplines including general, developmental, cardiology, emergency, behavioral, respirology, neonatal intensive care, and community. The pediatricians agreed with all of the usefulness questions, indicated by median ratings of 3 (agree) or 4 (strongly agree) (Table 4). Only one pediatrician was dissatisfied with the response options, and one was dissatisfied with the order of the questions, suggesting a shifting of sections. Ten were dissatisfied with PEHH length, and 11 were dissatisfied with the anticipated time for completion, indicated by a median rating of 2 (dissatisfied) for both questions (Table 4).

\section{Discussion}

After two rounds of a modified Delphi technique involving pediatric environmental health experts, excellent consensus and overall agreement was achieved for question relevancy and comprehensiveness, respectively. Gathering usability and usefulness feedback from P/G and pediatricians further yielded a discrepancy in their views on the PEHH length and the time/anticipated time for administration. P/G are generally satisfied with the PEHH length and their devoted time, 
Table 3. Median ratings for each usability survey question, by condition category.

Please indicate your level of agreement for the following questions: (1=Strongly disagree, 2=Disagree, 3=Agree, 4=Strongly Agree)

\begin{tabular}{|c|c|c|c|}
\hline & Other $(n=20)$ & Respiratory $(\mathrm{n}=11)$ & Neurodevelopmental $(n=14)$ \\
\hline & $\begin{array}{l}\text { Median Rating } \\
\text { (Range) }\end{array}$ & Median Rating (Range) & Median Rating (Range) \\
\hline 1. The questionnaire did a good job of exploring your and your child's environment. & $(2-4)$ & $(3-4)$ & $(2-4)$ \\
\hline 2. The questionnaire allowed you to learn about your and your child's environment. & $(2-4)$ & $(2-4)$ & $(2-4)$ \\
\hline 3. The questions were easy to understand. & $(3-4)$ & $(3-4)$ & $\left(3 \frac{3}{-4}\right)$ \\
\hline 4. The questions were easy for you to answer. & $\left(3-\frac{3}{-4}\right)$ & $(3-4)$ & $(3-4)$ \\
\hline 5. The order of the questions flowed well. & $(3-4)$ & $(3-4)$ & $\left(3-\frac{3}{-} 4\right)$ \\
\hline 6. The response options were appropriate for this questionnaire. & $(2-4)$ & $(3-4)$ & $\left(3-\frac{3}{-} 4\right)$ \\
\hline 7. The environment is an important influence in your and your child's health. & $(3-4)$ & $(3-4)$ & $(3-4)$ \\
\hline \multicolumn{4}{|c|}{ Please indicate your level of satisfaction for the following questions: How satisfied are you with: $(1=$ Very dissatisfied, $2=$ Dissatisfied, $3=$ Satisfied, $4=$ Very Satisfied $)$} \\
\hline 8. The length of the questionnaire. & $(2-4)$ & $\left(2 \frac{3}{-} 4\right)$ & $\left(3-\frac{3}{-4}\right)$ \\
\hline 9. The amount of time taken to complete the questionnaire. & $(2-4)$ & $(3-4)$ & $\left(3-\frac{3}{-} 4\right)$ \\
\hline
\end{tabular}

Table 4. Median rating for each PEHH "usefulness" question ( $\mathrm{n}=15$ pediatricians).

Please indicate your level of agreement for the following questions: (1=Strongly disagree, 2=Disagree, 3=Agree, 4=Strongly agree)

\begin{tabular}{|c|c|}
\hline & Median Rating (Range) \\
\hline 1. The PEHH is useful to explore the environment of children and their parents/guardians (P/G). & $\begin{array}{c}3 \\
(3-4)\end{array}$ \\
\hline 2. The PEHH can give $P / G$ more awareness about the environment and it's role in their child's health. & $\begin{array}{c}3 \\
(3-4)\end{array}$ \\
\hline 3. The questions are clear enough for pediatricians to ask. & $\begin{array}{c}3 \\
(3-4)\end{array}$ \\
\hline 4. The questions are clear enough for $P / G$ to understand. & $\begin{array}{c}3 \\
(2-4)\end{array}$ \\
\hline 5. The order of the questions flow well. & $\begin{array}{c}3 \\
(2-4)\end{array}$ \\
\hline 6. The response options are appropriate for the PEHH & $\begin{array}{c}3 \\
(2-4)\end{array}$ \\
\hline 7. The role of the environment in children's health is of great importance. & $\begin{array}{c}4 \\
(3-4)\end{array}$ \\
\hline 8. The PEHH is valuable to clinical pediatric practice. & $\begin{array}{c}3 \\
(3-4)\end{array}$ \\
\hline \multicolumn{2}{|c|}{ Please indicate your level of satisfaction. How satisfied are you with: (1=Very dissatisfied, $2=$ Dissatisfied, $3=$ Satisfied, $4=$ Very satisfied $)$} \\
\hline 9. The length of the PEHH & $\begin{array}{c}2 \\
(2-4)\end{array}$ \\
\hline 10. The amount of time anticipated to administer the PEHH. & $\begin{array}{c}2 \\
(1-4)\end{array}$ \\
\hline
\end{tabular}

whereas pediatricians found it to be lengthy and anticipate too much clinical burden.

The need for tools to accurately, evaluate environmental exposures in pediatric clinics makes our work substantially relevant. Although varieties of questionnaires are used in clinical settings, systematic reviews in various disciplines have identified few questionnaires as validated [27]. Questionnaires exploring environmental risk factors have been identified [9], but are limited in their measurement scope and more importantly; they have not been evaluated sufficiently, properly, or comprehensively, which could compromise the quality of the collected data. By applying the recommended Terwee (2007) criteria, our study undertook a fundamental step in establishing content validity of the Pediatric Environmental Health History (PEHH). Content validity is considered one of the most important validation steps and establishing other forms of validity prior to this cannot replace it [12]. Once content validity for a questionnaire has been established, other forms of validity must be assessed to strengthen its credibility for clinical use.

To achieve study objectives, we relied on a modified Delphi technique, an iterative approach commonly used to generate consensus from a panel of experts on a topic of interest $[17,18]$. The PA achieved in Delphi round 1 indicated high response subjectivity and need for content improvement. Although a recognized limitation in our Delphi method did not give the experts opportunity to vote on the rejected questions between rounds, our predetermined selection criteria helped to mitigate individual investigator biases. The degree of expert consensus in conjunction with specific criteria for "excellent content validity" further delivered strength to our approach $[17,18]$. The achieved level of convergence was also used as a measure of content 
improvement and facilitated our determination of difficult areas between rounds. Although excellent expert consensus was achieved after round 2, the experts did identify areas needing minor clarity and re-formatting. Despite attending to their minor requests, we felt that a final round of review was not mandatory provided the nature of these suggestions.

In identifying potential Delphi subjects, limitations in the available data repository prevented the identification of the total number of global environmental health experts. This forced us to rely on established clinical contacts that were affiliated with the WHO, and mainly PEHSU members, which may have caused some selection bias. As children's environmental health is an emerging discipline, a precise data directory of experts appears to be unavailable. Previous studies also indicate that pediatricians are largely unaware of the PEHSUs and that hardly any refer their patients to the specialty [6,7]. We further suspect that the number of pediatric environmental health experts on an international level is substantially low. Despite the low response rate, our inclusion criteria allowed us to ensure that our participating experts are credible and that they could contribute knowledge towards children's environmental health from varying angles.

The opposing views of $\mathrm{P} / \mathrm{G}$ and pediatricians are important determinants for the clinical applicability of the PEHH and represents an interesting finding in our study. Pediatricians expressed a similar attitude in previous studies indicating that the environmental history would in general take up too much time, despite their overall optimism towards environmental health [6,7]. Although the average time taken for $\mathrm{P} / \mathrm{G}$ to complete the PEHH was approximately 30 minutes, there was a large variation of 21 to 49 minutes. This variation could be explained by the fact that more complicated cases may necessitate more time for probing questions. The modes of questionnaire administration [29] must also be considered, as in reality, direct clinical interviews may take more time. This could explain why pediatricians might be less satisfied towards their anticipated time in conducting a thorough history. While we recognize the limitation in the response rate from pediatricians, we feel that perhaps many of them are reluctant to participate in commenting on a tool that they may be unfamiliar with or lack confidence in discussing, complementary to previous studies that assessed their confidence with respect to environmental health $[6,7]$. This suggests the need to promote Children's Environmental Health and increase awareness and training throughout all subspecialties.

\section{Conclusion}

The need for validated questionnaires is critical to clinical practice and various areas of health research. Children's environmental health is no exception. By following recommended guidelines, we established content validity for the $\mathrm{PEHH}$, ensuring a relevant and comprehensive set of questions to clinically explore environmental risk factors for children. Further validity is now ready to be established, exploring relationships between different sections and questions (construct validity), and determining the extent of error involved in the data collected (reliability). These necessary steps will further the evidence to help promote a routine clinical environmental screening on pediatric patients, which aligns with parents/guardians needs.

\section{Financial disclosure}

The authors declare that they do not have any financial relationships relevant to this article to disclose.

\section{Conflict of interest}

The author(s) declare that they have no competing interests.

\section{Acknowledgements}

Parneet Jaggi conceptualized, designed and conducted the study, drafted the initial manuscript. Alvaro Osornio-Vargas, Corresponding author, mentored and supervised Parneet Jaggi throughout the study phases and reviewed and revised the manuscript.

Irena Buka supervised Parneet Jaggi throughout the study phases, facilitated data collection at the main site, reviewed and revised the manuscript.

Jesus Serrano Lomelin facilitated the statistical analyses, reviewed and revised the manuscript. All authors approved the final manuscript as submitted and agree to be accountable for all aspects of the work.

The supervisory committee facilitated project development. Significant contributions were made by: Dr. Sunita Vohra, Department of Pediatrics, Complementary and Alternative Medicine; Dr. Ying Cui, Department of Education, Educational Psychology, and Dr. Irena Buka, Department of Pediatrics, Children's Environmental Health.

Study Phase I involved contribution from the international, Pediatric Environmental Health Experts associated with Pediatric Environmental Health Specialty Units (PEHSUs): Drs. Maria Jose Moll, Catherine Karr, Alan Abelsohn, Leslie Rubin, Mark Miller, Kevin Chatham-Stephens, Maida Galvez, Jennifer Lowry, Matt Karwowski, and Perry Sheffield. Without them, this study would not have been possible.

Study Phase II involved valuable contribution and feedback from parents/guardians of children attending the Child Health Clinic, Mother Rosalie Building, Misericordia Hospital, Edmonton AB, Canada.

The Women and Children's Health Research Institute's Clinical Research Informatics Core, University of Alberta, were of substantial help during data collection and management.

\section{Funding}

The Emerging Research Team Grant (ERTG) from the Faculty of Medicine and Dentistry, University of Alberta and Alberta Health Services, provided partial support for this study. The Hair Massacure Grant provided partial support for this study. The funding agencies were not involved in conducting the study. The Department of Pediatrics, University of Alberta provided support.

\section{References}

1. Clark B, Vandermeer B, Simonetti A, Buka I (2010) Is lead a concern in Canadian autistic children? Paediatr Child Health 15: 17-22. [Crossref]

2. Fromme H, Mosch C, Morovitz M, Alba-Alejandre I, Boehmer S, et al. (2010) Preand postnatal exposure to perfluorinated compounds (PFCs). Environ Sci Technol 44: 7123-7129. [Crossref]

3. Global plan of action for children's health and the environment 2011.

4. Garbutt JM, Leege E, Sterkel R, Gentry S, Wallendorf M, et al. (2012) What are parents worried about? Health problems and health concerns for children. Clin Pediatr (Phila) 51: 840-847. [Crossref]

5. Kilpatrick N, Frumkin H, Trowbridge J, Escoffery C, Geller R, et al. (2002) The environmental history in pediatric practice: a study of pediatricians' attitudes, beliefs, and practices. Environ Health Perspect 110: 823-827. [Crossref]

6. Trasande L, Boscarino J, Graber N, Falk R, Schechter C, et al. (2006a) The environment in pediatric practice: a study of New York pediatricians' attitudes, beliefs, and practices towards children's environmental health. J Urban Health 83: 760-772. [Crossref] 
7. Trasande L, Schapiro ML, Falk R, Haynes KA, Behrmann A, et al. (2006b) Pediatrician attitudes, clinical activities, and knowledge of environmental health in Wisconsin. WMJ 105: 45-49. [Crossref]

8. Roberts JR, Karr CJ, de Ybarrondo L, McCurdy LE, Freeland KD, et al. (2013) Improving pediatrician knowledge about environmental triggers of asthma. Clin Pediatr (Phila) 52: 527-533. [Crossref]

9. World Health Organization, Children's Environmental Health, 2013. The paediatric environmental history: Recording children's exposure to environmental health threats: A "green page" in the medical record.

10. Roberts J, Balk S, Etzel R, et al., n.d. Pediatric environmental history (0-18 years of age).

11. Texas Childhood Lead Poisoning Prevention Program, 2011. Risk assessment for lead exposure: Parent questionnaire.

12. Terwee CB, Bot SD, de Boer MR, van der Windt DA, Knol DL, et al. (2007) Quality criteria were proposed for measurement properties of health status questionnaires. $J$ Clin Epidemiol 60: 34-42. [Crossref]

13. World Health Organization, Children's Environmental Health, Capacity Building, 2013. Training modules and instructions for health care providers.

14. Jones J, Hunter D (1995) Consensus methods for medical and health services research. BMJ 311: 376-380. [Crossref]

15. Association of Occupational and Environmental Clinics, 2013. Pediatric environmental health specialty units.

16. World Health Organization. Children's environmental health, n.d.

17. Hsu C, Sanford B (2007) The delphi technique: Making sense of consensus 12: 1.

18. Adler M, Ziglio E (1996) Gazing into the oracle. Philadelphia: Jessica Kingsley.

19. Harris PA, Taylor R, Thielke R, Payne J, Gonzalez N, et al. (2009) Research electronic data capture (REDCap)--a metadata-driven methodology and workflow process for providing translational research informatics support. J Biomed Inform 42: 377-381. [Crossref]

20. Polit DF, Beck CT (2006) The content validity index: are you sure you know what's being reported? Critique and recommendations. Res Nurs Health 29: 489-497. [Crossref]

21. Lynn MR (1986) Determination and quantification of content validity. Nurs Res 35: 382-385. [Crossref]

22. Mokkink LB, Terwee CB, Patrick DL, Alonso J, Stratford PW, et al. (2010) The COSMIN study reached international consensus on taxonomy, terminology, and definitions of measurement properties for health-related patient-reported outcomes. $J$ Clin Epidemiol 63: 737-745. [Crossref]

23. Carlsson N, Alehagen S, Andersson Gäre B, Johansson A (2011) "Smoking in Children's EnvironmentTest": a qualitative study of experiences of a new instrument applied in preventive work in child health care. BMC Pediatr 11: 113. [Crossref]

24. Kreuger R, Casey M (2009) Focus groups: A practical guide for applied research London: SAGE publications Ltd.

25. Morgan D (1997) Focus groups as qualitative research 2nd ed. Newbury Park, CA: Sage Publications Inc.

26. Sandelowski M (1995) Sample size in qualitative research. Res Nurs Health 18: 179183. [Crossref]

27. Reid GT, Walter FM, Brisbane JM, Emery JD (2009) Family history questionnaires designed for clinical use: a systematic review. Public Health Genomics 12: 73-83. [Crossref]

28. Toupin April K, Moher D, Stinson J, Byrne A, White M, et al. (2012) Measurement properties of questionnaires assessing complementary and alternative medicine use in pediatrics: A systematic review. PLoS One 7: e39611.

29. Bowling A (2005) Mode of questionnaire administration can have serious effects on data quality. J Public Health (Oxf) 27: 281-291. [Crossref]

Copyright: $@ 2016$ Jaggi P. This is an open-access article distributed under the terms of the Creative Commons Attribution License, which permits unrestricted use, distribution, and reproduction in any medium, provided the original author and source are credited. 